\title{
PROFUNDIDAD DE SIEMBRA Y TAMAÑO DE LAS SEMILLAS: DESARROLLO DE LAS PLÁNTULAS DE FRIJOL MUNGO
}

\author{
SOWING DEPTH AND SEED SIZE ON MUNG \\ BEAN SEEDLING PERFORMANCE
}

\section{${ }^{1}$ Adriene Vieira de Faria, ${ }^{2}$ Kássia de Paula Barbosa, ${ }^{3}$ Adriana Rodolfo da Costa, ${ }^{4}$ Patricia Costa Silva, ${ }^{5}$ Anailda Angélica Lana Drumond, 6 Janaína Borges de Azevedo França, ${ }^{7}$ Gilzângela Martins Silva}

\author{
1,7 Ingeniara Agrícola, Universidad Estadual de Goiás, Unidad Universitaria Santa Helena de Goiás, \\ Santa Helena de Goiás, Goiás, Brasil. ${ }^{2,5}$ Doctor en Ciencias Agrícolaş Instituto Federal Goiano, Campus Rio \\ Verde, Rio Verde, Goiás, Brasil. 3, 4 Doctor en Agronomía, Universidad Estadual de Goiás, Unidad \\ Universitaria Santa Helena de Goiás, Santa Helena de Goiás, Goiás, Brasil. \\ ${ }^{6}$ Doctor en Ciencias Agrarias, Universidad Estadual de Goiás, Unidad Universitaria de Ipameri, Ipameri, Goiás, Brasil.

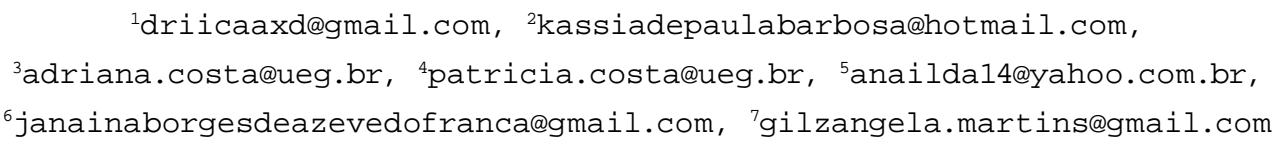

Citación: Faria, A.V., Barbosa, K.P., Costa, A.R., Silva, P.C., Drumond, A.A., França, J.B., y Silva, G.M. (2021). Profundidad de siembra y tamaño de las semillas: desarrollo de las plántulas de frijol Mungo. Revista de Investigación Agraria y Ambiental, 12(2), 13 - 24. DOI: https://doi.org/10.22490/21456453.3833

\section{RESUMEN}

Contextualización del tema: el frijol mungo es ampliamente cultivado en Asia y en Brasil, y ha surgido como una opción para el cultivo en el segundo cultivo en el Centro Oeste de Brasil, especialmente cuando se pretende la rotación con pastos; sin embargo, la aparición desigual y lenta de las plántulas de frijol mungo puede conducir a retrasos en el desarrollo del cultivo y perjudicar la posició nal.

Vacío de investigación: algunas de las condiciones que afectan al establecimiento inicial del cultivo son la profundidad de siembra y el tamaño de la semilla. Estos factores todavía están siendo discutidos en el entorno cientí co, especialmente para una cultura recientemente insertada en Brasil.

Propósito del estudio: el objetivo fue evaluar el efecto del tamaño de las semillas y la estandarización de la profundidad de la siembra en el rendimiento de las plántulas de frijol mungo.

Metodología: el ensayo se llevó a cabo en un Latossuelo Rojo, con clima Aw, precipitación media anual de $1300 \mathrm{~mm}$ y distribución bimodal. El diseño experimental utilizado fue 
completamente aleatorio, en un esquema factorial de $2 \times 3$ con 8 réplicas de 25 semillas, con un total de 48 parcelas. Los factores analizados fueron de los tamaños de semilla (3 y $4 \mathrm{~mm}$ ) y tres profundidades de siembra $(1,2$ y $3 \mathrm{~cm})$. Se evaluó el índice de velocidad de emergencia (IVE), las plántulas no emergidas, las plántulas normales, la masa fresca, la masa seca y la longitud de las plántulas. Los datos se sometieron al análisis de varianza y, cuando se aplicaba una probabilidad signi cativa del $5 \%$, se aplicó una prueba de Tukey para comparar los medios de los factores en estudio.

Resultados y conclusiones: se veri có que, cuando se siembran a $1 \mathrm{~cm}$ de profundidad, las semillas de frijol mungo tienen IVE más bajo; sin embargo, obtuvieron un menor porcentaje de plántulas no relevantes para el estudio, y un mayor porcentaje de plántulas normales emergió a una profundidad de $3 \mathrm{~cm}$. Por lo tanto, la profundidad más recomendada para la siembra de frijoles mungo fue de 2 $\mathrm{cm}$, independientemente del tamaño de la semilla utilizada. Lo tamaño de semilla de $4 \mathrm{~mm}$ permitió el desarrollo de plántulas con mayor acumulación de materia seca.

Palabras-clave: emergencia de plántulas; índice de velocidad de emergencia; plántulas normales; Vigna radiata (L) Wilczek

\section{ABSTRACT}

Contextualization: The mung bean is widely cultivated in Asia and Brazil, it has emerged as an option for cultivation in the second crop in the Central West Brazil, especially rotation with grasses is intended; however, the uneven and slow emergence of mung bean seedlings can lead to delays in the development of the crop and impair th nal stand.
Knowledge gap: Some of the conditions that affect the initial establishment of the crop are the sowing depth and the size of the seed. These factors are still under discussion in the scienti c environment, especially for a culture recently introduced in Brazil.

Purpose: the objective was to evaluate the effect of seed size and sowing depth standardization on the performance of mung bean seedlings.

Methodology: The trial was conducted in a Red Latosol, with Aw climate, average annual rainfall of $1300 \mathrm{~mm}$ and bimodal distribution. The experimental design used was completely randomized, in a $2 \times 3$ factorial scheme with 8 replicates of 25 seeds, totaling 48 plots. The factors analyzed were two seed size ( 3 and 4 $\mathrm{mm}$ ) and three sowing depths $(1,2$ and $3 \mathrm{~cm})$. The emergency speed index (ISE), non-emerged seedlings, normal seedlings, fresh mass, dry mass and seedling length were evaluated. The data were submitted to variance analysis and, when signi cant at $5 \%$ probability, a Tukey test was applied to compare the means of the factors under study.

Results and conclusions: It was veri ed that, when sown at $1 \mathrm{~cm}$ depth, the seeds of mung bean have lower ISE, however, they obtained a lower percentage of seedlings not relevant for the study, and a higher percentage of normal seedlings emerged than at a depth of $3 \mathrm{~cm}$. Thus, the most recommended depth for the sowing of mung beans was $2 \mathrm{~cm}$, regardless of the size of the seed used. The seed class of 4 $\mathrm{mm}$ allowed the development of seedlings with higher dry matter accumulation.

Keywords: Emergency speed index; Normal seedlings; Seedling emergence; Vigna radiata (L) Wilczek; 


\section{RESUMEN GRÁFICO}

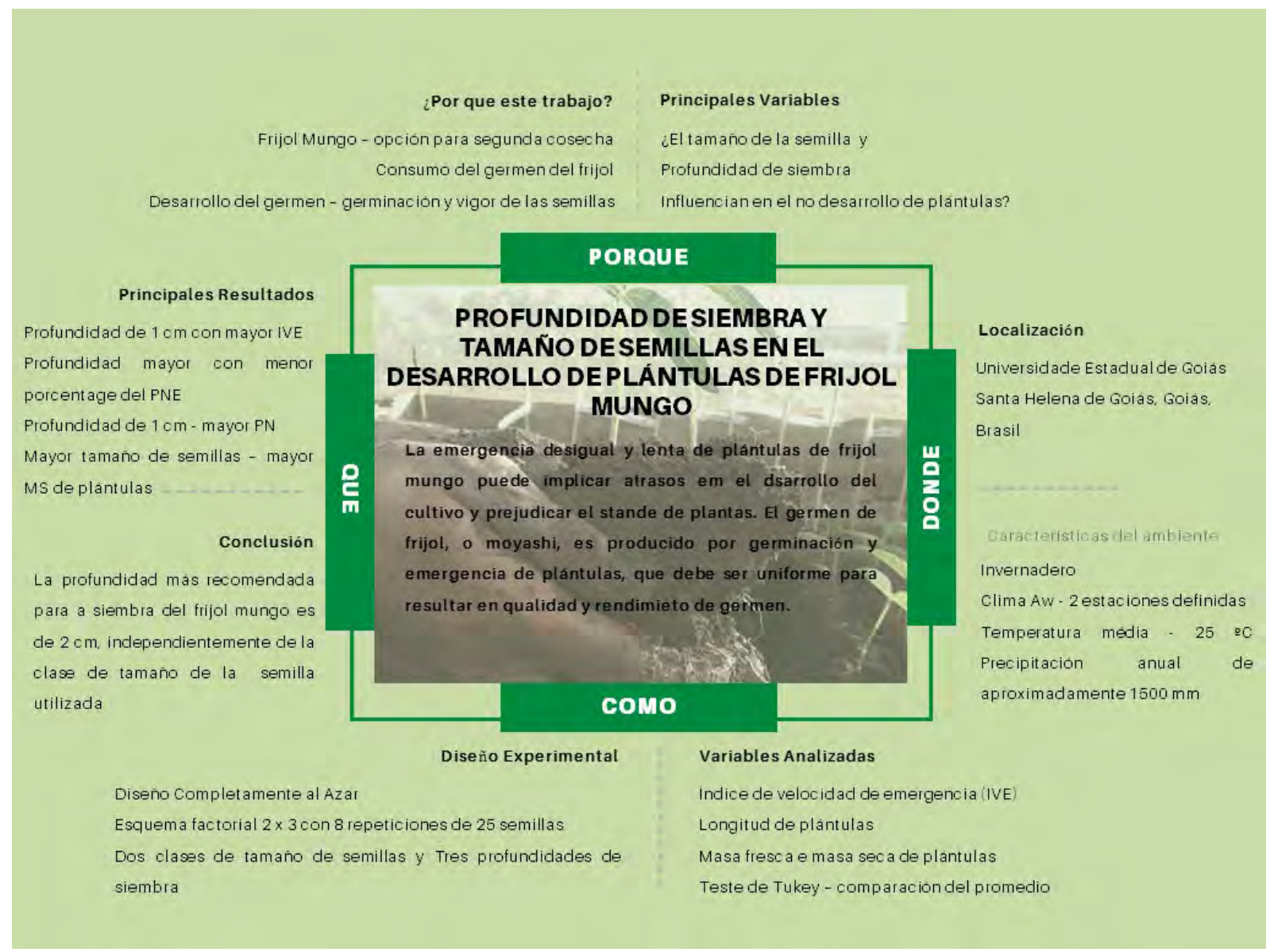

Fuente: autores

\section{INTRODUCCIÓN}

En Brasil, la producción total de frijol en la cosecha 2018/19 fue de 3,02 millones de toneladas, de los cuales 1,88 millones de toneladas son de frijol de colores (Phaseolus vulgaris L.), 497 mil toneladas de frijol común negro (Phaseolus vulgaris L.) y, 638 mil toneladas de frijol Caupi (Vigna unguiculata [L.] Walp.) (Conab, 2019). El frijol es una fuente económica de proteínas, carbohidratos y bras, además de eso, contiene poca grasa. En la dieta del ser humano se incluye para bene cios nutricionales, especialmente en micronutrientes (Yu-Wei y Wang, 2015).

El frijol Mungo (Vigna radiata [L.] Wilczek) es una leguminosa cultivada en muchas regiones del mundo, especialmente en el continente Asiático (Dikshit et al., 2017). En Brasil, surgió como una buena opción para sembrar en áreas de cultivo de segunda cosecha en diversas regiones del Centro Oeste, especialmente por presentar un ciclo de maduración corto, permitir cosecha mecanizada y ser una opción de exportación para países asiáticos (Farias Neto et al., 2019). Igualmente es una excelente opción en la rotación con pastos, como maíz y trigo (Dahiya et al., 2015). Asociado a lo anterior, aumenta el consumo del brote del frijol, conocido como Moyashi, que posee elevada calidad nutricional (Vieira et al., 2011). El brote es producido por germinación de las semillas y queda listo para consumo de 3 a 7 días 
aproximadamente (Silva et al., 2013). El desarrollo del brote depende de la germinación y vigor de las semillas, por eso la calidad debe ser fundamental para garantizar la productividad del frijol mungo (Vieira et al., 2003).

La uniformidad del tamaño de la semilla es una estrategia para mejorar la emergencia de plántulas, dado que las semillas mayores o con mayores densidades pueden poseer un embrión bien formado y mayor cantidad de reserva, lo que hace que sean más vigorosas (Carvalho y Nakagawa, 2012). Steiner et al. (2019) a rmaron que el tamaño de la semilla establece una importante evidencia de calidad siológica, pues puede afectar la germinación y el crecimiento de plántulas, especialmente en condiciones estresantes. No obstante, hay evidencia de que puede ocurrir lo opuesto, es decir, que el tamaño de la semilla no afecte el vigor y germinación del frijol común (Mondo et al., 2014).

Otro factor que in uye en la emergencia de la plántula es la profundidad de siembra. La profundidad en que se deposita la semilla está ligada directamente a la calidad de la geminación y desarrollo de la planta (Silva et al., 2008). Siembras más profundas, en suelo húmedo y en bajas temperaturas favorecen hongos del suelo, que provocan daños en semillas, además de aumentar el gasto de energía de la plántula para emerger (Munkvold, 2003). Cuando se siembra en profundidades reducidas, se propicia el ataque de plagas (Jeller y Perez, 1997) o predispone al estrés térmico y al dé cit hídrico, pues la semilla necesita estar cubierta por el suelo húmedo para que el proceso de remojo ocurra (Alves et al., 2014).

No está claro efecto del tamaño de las semillas sobre la calidad siológica, especialmente en lo que corresponde al frijol mungo.
Aún hay mucho interés por esclarecer ese cuestionamiento y por obtener conocimiento sobre si existe una relación entre el tamaño y calidad de las semillas, y si esa relación permanece cuando la siembra ocurre en diferentes profundidades. Por esto, existe la necesidad del estudio de nuevas estrategias que se puedan sugerir, especialmente a los pequeños productores. Teniendo en cuenta lo anterior, independientemente del genotipo, el objetivo fue analizar el efecto de la estandarización del tamaño de las semillas y profundidad de siembra en el desarrollo de plántulas de frijol mungo.

\section{MATERIAL Y MÉTODOS}

El estudio se realizó en la Universidade Estadual de Goiás (Unidad Universitaria Santa Helena de Goiás), en el Sureste Goiano. El área se localizada a 17048'49" S y 50³5'49" W, a 595 m.s.n.m.. La región posee clima clasi cado como Aw, que presenta una estación más seca, no invierno (Alvares et al. 2013). El experimento se realizó en agosto de 2018, período sin precipitaciones en la región, y con temperatura mínima de $20^{\circ} \mathrm{C}$ y máxima de $32^{\circ} \mathrm{C}$. El experimento se llevó a cabo en un área bajo una pantalla de sombra al $50 \%$, en Latossuelo Rojo Distró co. El agua durante la prueba se suministró mediante microaspersores para mantener la humedad del suelo cerca de la capacidad de campo.

Se utilizó un diseño experimental completamente aleatorizado, en esquema factorial 2 $\times 3$ con 8 repeticiones de 25 semillas, para un total de 48 porciones. Los factores analizados fueron los tamaños de semillas ( 3 y 4 $\mathrm{mm})$ y tres profundidades de siembra $(1,2$ y $3 \mathrm{~cm}$ ). Las semillas se clasi caron mediante el cierne con tamices circulares de 3 y $4 \mathrm{~mm}$ de diámetro. Se evaluaron las siguientes características: 
Índice de velocidad de emergencia (IVE): se observó diariamente la emergencia de las plántulas en un horario de nido (entre 9 y 10h de la mañana) hasta su estabilización, de acuerdo con Koch et al. (2015). Se contó el número de plántulas emergidas por día, dividendo ese número por la cantidad de días transcurridos desde la fecha de siembra; posteriormente, se calculó el índice de velocidad de emergencia de acuerdo con la ecuación 1 propuesta por Maguire (1962).

\section{IVE $=(E 1 / N 1)+(E 2 / N 2)+\ldots+(E n / N n)$ (ecuación 1)}

Donde:

IVE = índice de velocidad de emergencia; E1, $\mathrm{E} 2, \ldots, \mathrm{En}=$ número de plántulas normales emergidas computadas en el primer conteo, en el segundo conteo y en el último conteo; $\mathrm{N} 1, \mathrm{~N} 2, \ldots, \mathrm{Nn}=$ número de días de siembra hasta el primer conteo, hasta el segundo conteo y hasta el último conteo.

Se evaluó el porcentaje de emergencia de plántulas normales (PN) al I del análisis del IVE. Se consideraron como plántulas normales aquellas capaces de desarrollarse y originar una planta normal con todas estructuras esenciales bien crecidas, como el sistema de raíz, la parte aérea y las hojas primarias en expansión. Según las Reglas para Análisis de Semillas (RAS) (Brasil, 2009), una plántula normal es aquella que demuestra la capacidad para originar una planta normal; que presente el sistema de raíz vigoroso, la parte aérea bien crecida, número especí co de cotiledones, hojas primarias verdes en expansión, yema apical y coleóptilo (Brasil, 2009). Las demás se consideraron plántulas no emergidas (PNE).
Longitud de plántulas (LP): tras la estabilización de la emergencia (eso después de once días de haber sembrado), se analizaron 12 plántulas por sección, se midió la longitud de la parte aérea y raíz, considerando las plántulas normales, y se registraron los resultados en centímetros (cm) (Nakagawa, 1999).

\section{Masa fresca (MF) y masa seca (MS) de} plántulas: se utilizaron 12 plántulas normales. Tras la estabilización de la emergencia, estas plántulas se pesaron para adquirir datos de masa fresca. Después, se depositaron las plántulas de cada sección en costales de papel y se llevaron a secar en un invernadero, a una temperatura de $65^{\circ} \mathrm{C}$, durante $72 \mathrm{~h}$. Después de este periodo, se pesaron las muestras y se registraron los resultados en g plántula-1, según Nakagawa, (1999).

\subsection{ANÁLISIS ESTADÍSTICO}

Los datos obtenidos en cada variable fueron sometidos a pruebas de normalidad mediante el método de Shapiro-Wilk y de homogeneidad de varianzas mediante la prueba de Levene, utilizando el programa estadístico PAST. Después de comprobar los supuestos, se realizó un análisis de varianza asociado con la prueba $\mathrm{F} y$, cuando el nivel de probabilidad fue del $5 \%(P<0,05)$ se aplicó el test de Tukey $(p<0,05)$ con el $n$ de saber si existían diferencias signi cativas entre las medias de los factores de tratamiento (profundidad de siembra y tamaño de las semillas) y sus interacciones para cada variable, utilizando el programa estadístico SISVAR (Ferreira, 2011). Adicionalmente, se estudió la relación entre las variables relacionadas con el desarrollo de las plántulas de frijol mungo mediante un análisis de correlación de Pearson a nivel de probabilidad del $5 \%(P<0,05)$, utilizando el programa estadístico PAST. 


\section{RESULTADOS Y DISCUSIÓN}

La tabla 1 presenta los valores de F provenientes del análisis de varianza para las variables respuestas: Índice de Velocidad de Emergencia (IVE), Plántulas no emergidas (PNE), Plántulas normales (PN), Masa seca (MS), Masa fresca (MF) y longitud de plántula (LP) del frijol Mungo cultivado, en diferentes profundidades y tamaños de semillas. El factor profundidad presentó diferencias signi cativas para el IVE, PNE y PN. Sin embargo, el factor del tamaño de las semillas es signi cativo solo para la MS de las plántulas de frijol mungo. No ocurrió una interacción signi cativa entre la profundidad de siembra y tamaño de semillas para ninguna de las variables.

Tabla 1. Valores de F para Índice de Velocidad de Emergencia (IVE), Plántulas no emergidas (PNE), Plántulas normales (PN), Masa seca (MS), Masa fresca (MF) y longitud de plántula (LP) de frijol mungo cultivado en diferentes profundidades $(P)$ y tamaño de semillas $(C)$.

\begin{tabular}{l|c|c|c|c|c|c|c|}
\hline Fuente de variación & GL & IVE & PNE & PN & MS & MF \\
\hline Profundidad (P) & 2 & $7,987^{* *}$ & $3,565^{*}$ & $3,565^{*}$ & $0,345^{\mathrm{ns}}$ & $1,384^{\mathrm{ns}}$ & $1,39^{\mathrm{ns}}$ \\
\hline Tamaño (T) & 1 & $0,098^{\mathrm{ns}}$ & $0,040^{\mathrm{ns}}$ & $0,040^{\mathrm{ns}}$ & $41,540^{* *}$ & $0,77^{\mathrm{ns}}$ & $0,786^{\mathrm{ns}}$ \\
\hline P $\times$ T & 2 & $0,856^{\mathrm{ns}}$ & $0,499^{\mathrm{ns}}$ & $0,499^{\mathrm{ns}}$ & $1,019^{\mathrm{ns}}$ & $1,302^{\mathrm{ns}}$ & $1,265^{\mathrm{ns}}$ \\
\hline Resíduo & 43 & - & - & - & - & - \\
\hline CV (\%) & - & 18,01 & 26,56 & 26,56 & 11,11 & 9,53 \\
\hline
\end{tabular}

GL: Grados de Libertad y CV: Coe ciente de Variación. ${ }^{* *}{ }^{*}{ }^{*}{ }^{\text {ns }}$ - Signi cativo a 1 y a $5 \%$ y no signi cativo, respectivamente, por la prueba de F.

Fuente: Autores.

De acuerdo con la gura 1, las profundidades de 2 y $3 \mathrm{~cm}$ no se diferenciaron entre sí, y a profundidad de $1 \mathrm{~cm}$ se obtuvo un menor desempeño para IVE. Biezus et al. (2017) evaluaron diferentes cultivares de cuatro grupos comerciales (Cavalo, Jalo, Carioca y Preto) de frijol comunes (Phaseolus vulgaris L.) y encontraron que la deposición de la semilla a $2 \mathrm{~cm}$ de profundidad, en un Nitossolo-Latosol, es la más adecuada para los grupos comerciales evaluados, y cuando supera los $2 \mathrm{~cm}$, afecta negativamente a la emergencia y el índice de velocidad de emergencia. Estos autores atribuyeron ese decrecimiento a las condiciones en que se encuentra la semilla, como temperatura, humedad, calor y ataque de plagas. De esta forma, estos resultados concuerdan con los encontrados en este trabajo con frijol Mungo, donde la profundidad de $1 \mathrm{~cm}$ expuso las semillas a condiciones de microclima en la super cie del suelo; a esta profundidad, la temperatura y humedad tuvieron mayor incidencia en la planta. Se percibió que, a menor profundidad, el suelo se seca más rápido y queda con menor proporción de agua; esto in uye en el proceso de in Itración de agua en el suelo, así como en la temperatura que calienta la camada del suelo y evapora el agua. 


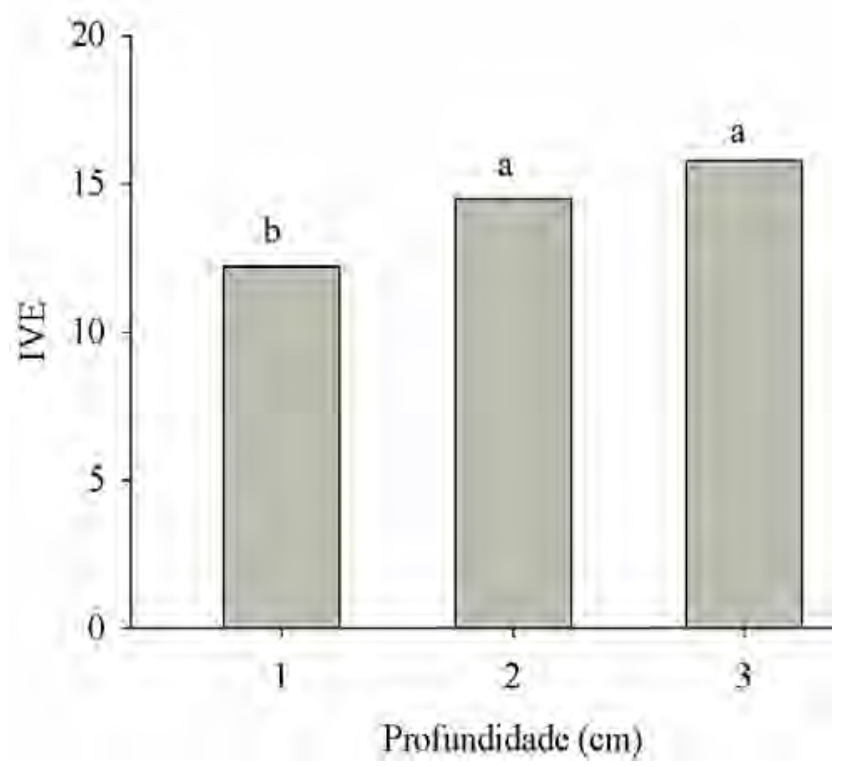

Figura 1. Índice de Velocidad de Emergencia (IVE) de plántulas de frijol Mungo en función de diferentes profundidades de siembra en Latossuelo Rojo. Diferencia mínima signi cativa (DMS: 2,19). Fuente: autores

En la gura 2, se puede percibir que la pro- se puede justi car por el mayor gasto enerfundidad de $1 \mathrm{~cm}$ produjo un menor porcen- gético que las plántulas sembradas a mayotaje de plántulas no emergidas en compara- res profundidades tuvieron para llegar a la ción con la profundidad de $3 \mathrm{~cm}$. Este hecho super cie del suelo.

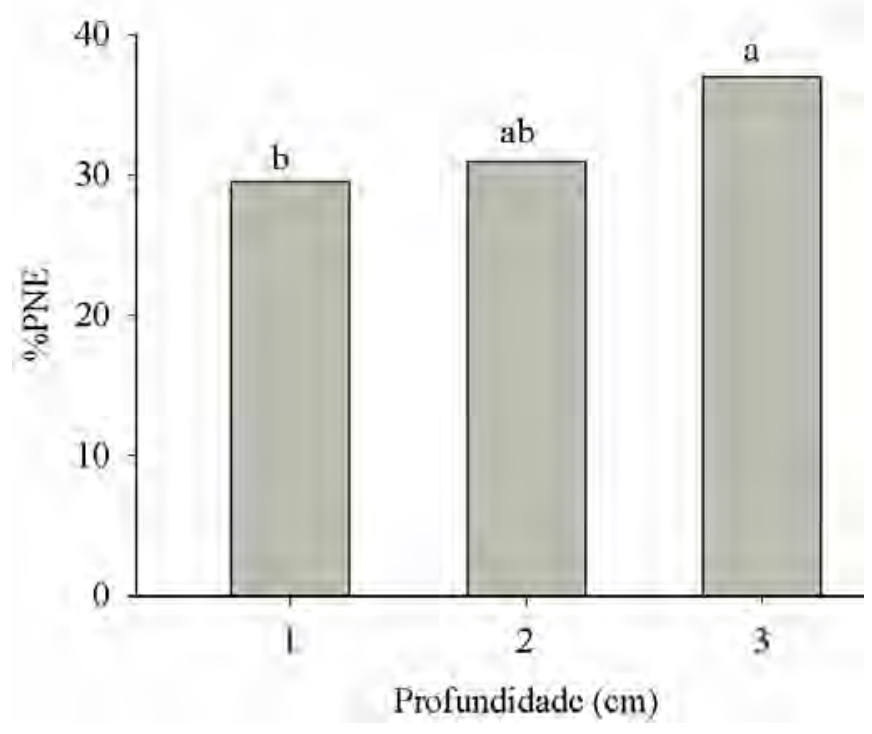

Figura 2. Porcentaje de plántulas no emergidas (PNE) de frijol mungo en función de diferentes profundidades de siembra en Latossuelo Rojo. Diferencia mínima signi cativa (DMS: 7,39\%). Fuente: autores 
De acuerdo con los resultados registrados en la gura 3, las profundidades de 1 y $2 \mathrm{~cm}$ no se diferenciaron entre ellas. No obstante, a $3 \mathrm{~cm}$ hubo un menor desempeño para el porcentaje de plántulas normales, comparadas a aquellas sembradas de manera más super cial. Resultados similares para emergencias también fueron encontrados por Pedó et al. (2014), donde se redujo la emergencia de plántulas (PN) con el aumento de la profundidad de siembra de frijoles, atribuyendo esta reducción a la mayor resistencia física impuesta por las capas suelo más denso, lo que proporciona una acción estresante en el desarrollo de la plántula y, consecuentemente, mayor porcentaje de plántulas no emergidas (PNE). Según Biezus et al. (2017), entre mayor sea la capa de suelo que tenga que romper la plántula, aumenta la deposición de semillas debido a que demanda mayor gasto energético de las reservas almacenadas en la semilla; esto afecta negativamente el rápido establecimiento de plántulas en el campo y la uniformidad inicial de las plántulas (Pedó et al., 2014).

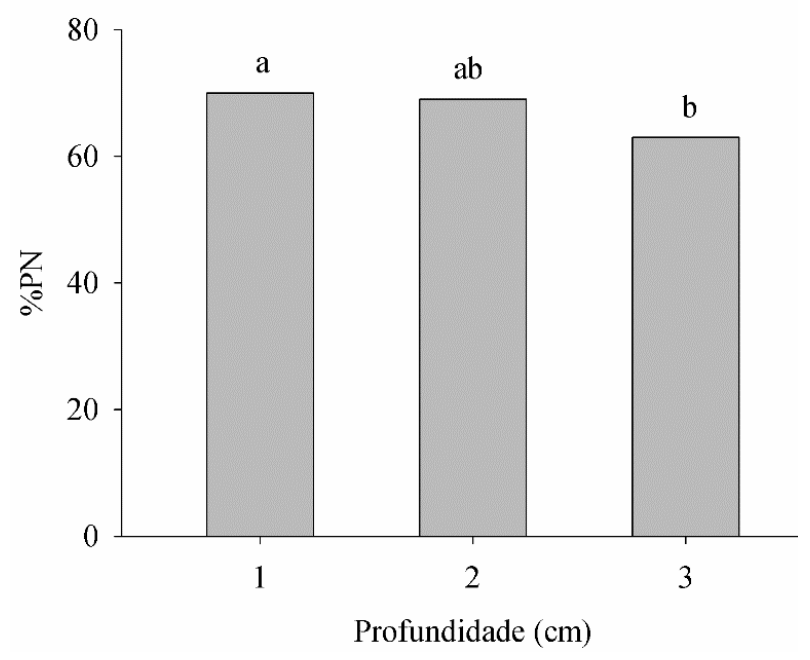

Figura 3. Porcentaje de plántulas normales (PN) de frijol mungo sembradas en diferentes profundidades en Latossuelo Rojo. Diferencia mínima signi cativa (DMS: 7,39\%). Fuente: autores
En la gura 4 se puede notar que el mayor tamaño de semilla $(4 \mathrm{~mm})$ presentó mejor desempeño en relación a la MS de plántulas de frijol Mungo. Las semillas más grandes o de mayor densidad son aquellas que normalmente tienen embriones bien formados y con mayores cantidades de reservas (Carvalho y Nakagawa, 2012), siendo potencialmente las que producirán plántulas más vigorosas. Carvalho y Nakagawa (2012) describieron que el tamaño de la semilla no siempre afecta la germinación, pero in uye en el vigor de la plántula. En consecuencia, las semillas de mayor tamaño generalmente causan un mejor desarrollo en las plántulas; sin embargo, se ha observado que las semillas mayores poseen embriones bien formados y con mayor cantidad de reserva de tejido, por lo tanto, son más vigorosas (Albuquerque et al., 2018); este factor también se observó en el presente trabajo, de acuerdo con lo descrito anteriormente.

Sin embargo, Pereira et al. (2013) a rmaron que, para el cultivo de soya bajo condiciones favorables de humedad, las semillas de mayor tamaño tienen mejor calidad siológica y producen plántulas más robustas, en comparación con las semillas de menor tamaño. Perin et al. (2002) observaron que semillas de tres cultivares de frijol de mayor tamaño poseían altura de plantas y biomasa superiores, sugiriendo que las mismas proporcionan un desarrollo inicial más vigoroso. El tamaño de la semilla está ligado a un mayor crecimiento y productividad del cultivo, lo que fomenta procesos metabólicos capaces de garantizar un buen desarrollo de plántulas (Pádua et al., 2010). 


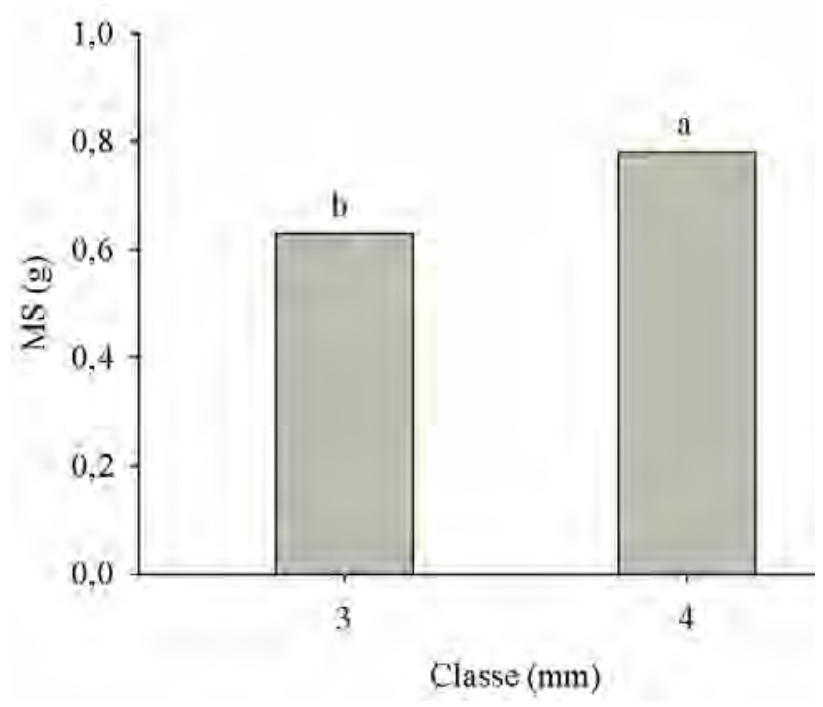

Figura 4. Masa Seca (MS) de Plántulas de frijol mungo en función de diferentes tamaños de semillas (clase). Diferencia mínima signi cativa (DMS: 0,005 g).

Fuente: autores

Mondo et al. (2014) percibieron que las pruebas de vigor basadas en el crecimiento de plántulas indicaron un probable efecto del tamaño en el desarrollo inicial de las plántulas de frijol a partir de semillas grandes. Pero, de acuerdo con estos autores, el tamaño de las semillas de frijol común no parece afectar la siología de la semilla, considerando que independientemente del tamaño, estas presentaron alto poder germinativo; sin embargo, incluso con el mismo poder germinativo, las plántulas de las semillas más grandes (4 mm) mostraron mayor acumulación de masa seca.
La gura 5 muestra las correlaciones de Pearson entre las variables relacionadas con el desarrollo de las plántulas de frijol Mungo. Se percibe una correlación fuerte y positiva entre IVE y PN $(r=0,84)$, y una correlación negativa entre el IVE y PNE $(r=-0,84)$. Lo anterior muestra que IVE está directamente relacionado con el desarrollo de plántulas normales. La MS de plántulas de frijol Mungo también resultó ser un factor signi cativo y positivo respecto a las variables IVE $(r=0,41)$ y PN $(r=0,36)$, pero con menor intensidad. Es decir, las plántulas normales (PN) de frijol Mungo también tuvieron una mayor acumulación de materia seca y un mayor índice de velocidad de emergencia. Es necesario tener en cuenta que estas variables, IVE, PN, PNE y MS, fueron determinantes para diferenciar las profundidades de siembra (Figura 1,2 y 3) y el tamaño de semillas de frijol mungo (Figura 4).

Amaro et al. (2015) también encontró una correlación signi cativa entre la masa seca de las plántulas mediante otras pruebas de evaluación la calidad siológica de la semilla de frijol común cV. Madrepérola, como IVE, LP, MF. Estos autores sugieren que, por lo tanto, la MS de plántulas es una prueba sensible para diferenciar lotes de semillas, siendo prometedora para evaluar la calidad siológica de las semillas de frijol común. De la misma forma, la MS de plántulas es un factor determinante para diferenciar tamaños de semillas de frijol Mungo. 


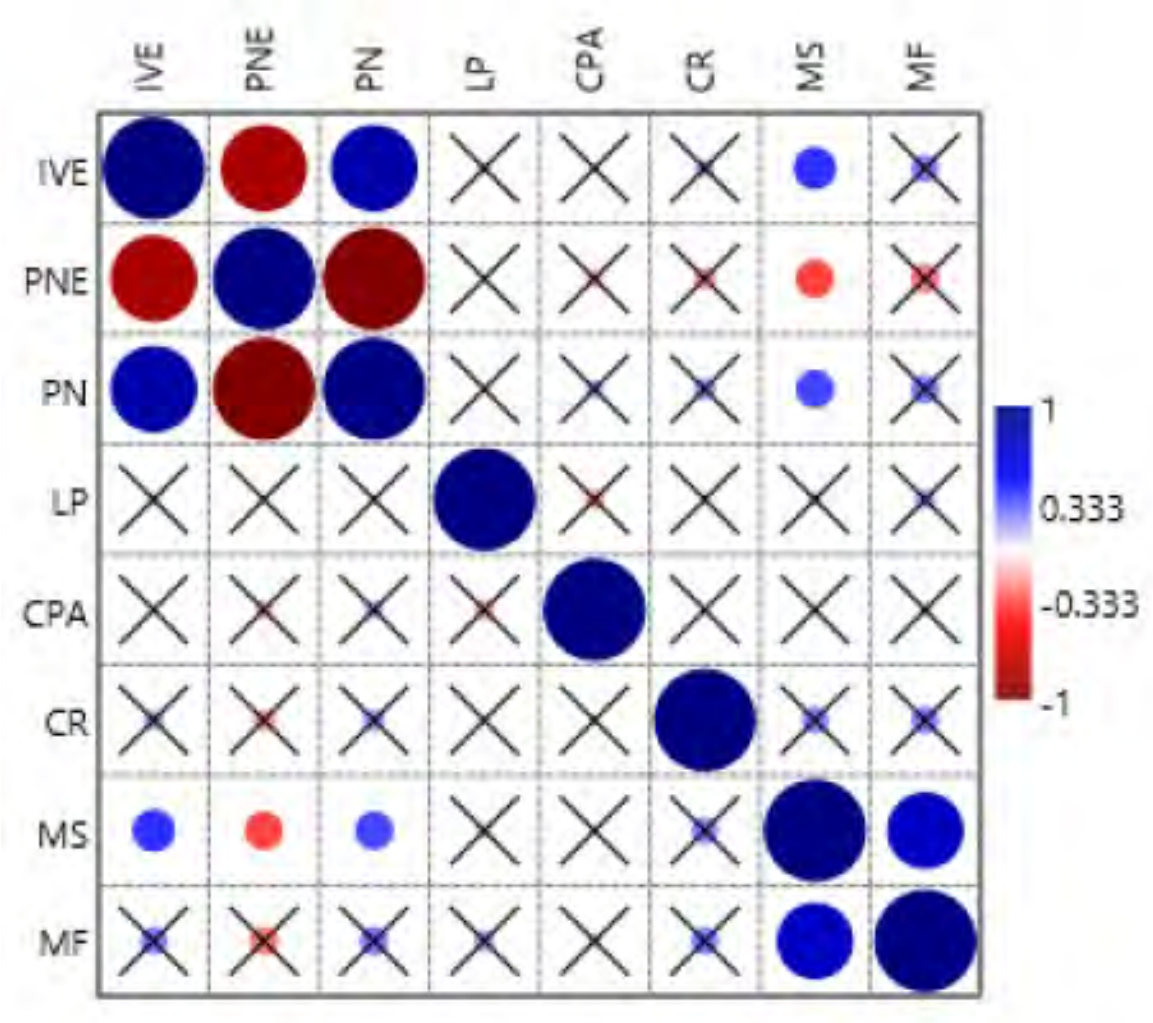

Figura 5. Correlación de Pearson entre las variables relacionadas con el desarrollo de las plántulas de frijol mungo. Índice de Velocidad de Emergencia (IVE), Plántulas no emergidas (PNE), Plántulas normales (PN), Masa seca (MS), Masa fresca (MF) y longitud de plántula (LP) de frijol mungo cultivado en diferentes profundidades y tamaños de semillas. " $X$ " indica correlación no signi cativa a nivel de $5 \%$ entre las variables. Fuente: autores

\section{CONCLUSIONES}

La siembra super cial de semillas de frijol Mungo limita el índice de velocidad de emergencia; sin embargo, a esta profundidad aumenta el porcentaje de plántulas normales.

La profundidad más recomendada para sembrar el frijol Mungo en estas condiciones es de $2 \mathrm{~cm}$, independientemente del tamaño de la semilla utilizada.

El tamaño de semilla de $4 \mathrm{~mm}$ posibilita el desarrollo de plántulas con mayor acúmulo materia seca.

\section{CONTRIBUCIÓN DE LA AUTORÍA}

Primer autor: Metodología, realizando el experimento de campo, investigación cientí ca, escritura - borrador original. Segundo autor: Investigación, conceptualización, análisis de datos, escritura - revisión. Tercer autor: Metodología, logística, investigación cientí ca, análisis de datos, escritura - revisión y edición. Cuarto autor: análisis de datos, revisión y edición. Quinto autor: Metodología, conceptualización, escritura - revisión y edición. Sexto autor: adquisición de materiales, escritura - revisión. Séptimo autor: Metodología, realizando el experimento de campo, escritura - revisión. 


\section{AGRADECIMIENTOS}

Al Departamento Administrativo, Universidad Estadual de Goiás, Unidad Universitaria de Santa Helena de Goiás (UEG), por el apoyo infraestructura y logística de la presente propuesta para desarrollar un semillero de investigación. Así como, estudiantes del curso de ingeniería agrícola que contribuyeron a la excepción del trabajo de campo. Y al profesor Nei Peixoto por poner a disposición semillas de frijol mungo.

\section{LITERATURA CITADA}

Albuquerque, K. A. D., Santos, G. J. S., Machado, M. A. B. L. (2018) In uência do tamanho das sementes na germinação de Caesalpinia ferrea Mart. ex Tul. var. Leiostachya Benth. Revista Ouricuri, 8(2):049-057, doi: https://doi. org/10.29327/ouricuri.v8.i2.a4

Alvares, C.A., Stape, J.L., Sentelhas, P.C., Gonçalves, J.L.M., Sparovek, G. (2013) Köppen's climate classi cation map for Brazil. Meteorologische Zeitschrift, 22(6):711-728. doi: https://doi. org/10.1127/0941-2948/2013/0507

Alves, A.U., Cardoso, E.A., Alixandre, T.F., Cavalcante, Í.H.L., Beckmann-Cavalcante, M.Z. (2014) Emergência de plântulas de fava em função de posições e profundidades de semeadura. Bioscience Journal, 30(1): 33-42.

Amaro, H.T.R., David, A.M.S.S., Assis, M.O., Rodrigues, B.R.A., Cangussú, L.V.S., Oliveira, M.B. (2015) Testes de vigor para avaliação da qualidade siológica de sementes de feijoeiro. $R e-$ vista de Ciências Agrárias 38(3): 383-389. doi: https://doi.org/10.19084/rca.16943

Biezus, A., Xavier, E., Brusamarello, A.P., Manteli, C., Carvalho, A.F.G. (2017) Emergência e desenvolvimento inicial de plântulas de feijão em diferentes profundidades

Biezus, A., Xavier, E., Brusamarello, A.P., Manteli, C., Carvalho, A.F.G. (2017) Emergência e desenvolvimento inicial de plântulas de feijão em diferentes profundidades de semeadura. Revista Brasileira de Ciências Agrárias, 12(4): 421-434. doi: https://doi.org/10.5039/ agraria.v12i4a5472

Brasil, Ministério da Agricultura, Pecuária e Abastecimento. (2009) Regras para análise de sementes. ed. 1, Brasília: Mapa/ACS, p. 161
Carvalho, N.M.; Nakagawa, J. (2012) Sementes: ciência, tecnologia e produção. 5. ed. Jaboticabal: FUNEP, 590 p.

CONAB, Companhia Nacional de Abastecimento (2019). Acompanhamento da safra brasileira grãos, Décimo Segundo levantamento Safra 2018/19. 6(12) p. 1-104.

Dahiya, P.K., Linnemann, A.R., Van Boekel, M.A.J.S., Khetarpaul, N., Grewal, R.B., Nout, M,J,R. (2015) Mung Bean: Technological and Nutritional Potential. Critical Reviews in Food Science and Nutrition, 55(5):670-688, doi: https://doi.org/10.1080/1040 8398.2012.671202

Dikshit, H.K., Kumari, J., Aski, M., Mishra, G.P., Singh, A. (2017) Outcrossing in Mungbean (Vigna radiata (L.) Wilczek) Depends on Cultivars and Seasons. Biotech Today, 7(1):82-85, doi: https:// doi.org/10.5958/2322-0996.2017.00014.X

Farias Neto, A.L., Nascimento, A.F., Rossoni, A.L., Magalhães, C.A.S., Ituassú, D.R., Hoogerheide, E.S.S., Ikeda, F.S., Fernandes Junior, F., Faria, G.R., Iserhangen, I., Vendurusculo, L.G., Morales, M.M., Carnevalli, R.A. (2019) Embrapa Agrossilvipastoril: Primeiras contribuições para o desenvolvimento de uma Agropecuária Sustentável 1Ed, Sinop (Brasil). Parte 8: Capítulo 16, Feijão-mungo como perspectiva para a safrinha em Mato Grosso, p. 635-641.

Ferreira, D.F. (2011) Sisvar: a computer statistical analysis system. Ciência e Agrotecnologia, 35(6):1039-1042, doi: https://doi.org/10.1590/ S1413-70542011000600001

Jeller, H., Perez, S.C.J.G. (1997) Efeito da salinidade de semeadura em diferentes profundidades na viabilidade e no vigor de Copaifera langsdorf Desf.- Caesalpiniaceae. Revista Brasileira de Sementes, 19(2):219-225.

Koch, F., Gehling, V.M., Pedó, T., Tunes, L.V.M., Villela, F.A., Aumonde, T.Z. (2015) Expressão do vigor de sementes e desempenho inicial de plantas de canola: efeito da profundidade de semeadura. $R e-$ vista de Agricultura, 90(2):193-201, doi: https:// doi.org/10.37856/bja.v90i2.195

Maguire, J.D. (1962) Speed of germination-aid selection and evaluation for seedling emergence and vigor. Crop Science, 2(2):176-177.

Mondo, V.H.V., Costa Neto, C.A., Costa, M.T.M., Nascente, A.S., Lacerda, M.C. (2014) Seed Size Does Not Affect Germination or Seed Vigor of Common Bean. Seed Technology, 36(1): 81-88, doi: https://www.jstor.org/stable/24642290 
Munkvold, G.P. (2003) Epidemiology of Fusarium diseases and their mycotoxins in maize ears. European Journal of Plant Pathology, 109(7):705-713, doi: https://doi.org/10.1023/A:1026078324268

Nakagawa, J. (1999) Testes de vigor baseados no desempenho das plântulas. In: KRZYZANOSKI, F. C.; VIEIRA, R. D.; FRANÇA NETO, J. B. (Ed.). Vigor de sementes: conceitos e testes. Londrina: ABRATES, p. 2.1-2.24.

Pádua, G.P.D., Zito, R.K., Arantes, N.E., França Neto, J.B. (2010) In uência do tamanho da semente na qualidade siológica e na produtividade da cultura da soja. Revista Brasileira de Sementes, 32(3):009-016, doi: https://doi.org/10.1590/ S0101-31222010000300001

Pereira, W.A., Pereira, S.M.A., Dias, D.C.M.S.F. (2013) In uence of seed size and water restriction on germination of soybean seeds and on early development of seedlings. Journal of Seed Science, 35(3):316-322, doi: https://doi. org/10.1590/S2317-15372013000300007

Pedó, T.; Segalin, S. R.; Silva, T. A.; Martinazzo, E. G.; Neto, A. G.; Aumonde, T. Z.; Villela, F. A. (2014) Vigor de sementes e desempenho inicial de plântulas de feijoeiro em diferentes profundidades de semeadura. Revista Brasileira de Ciências Agrárias, 9(1):59-64, doi: https://doi. org/10.5039/agraria.v9i1a3631

Perin, A., Araújo, A.P., Teixeira, M.G. (2002) Efeito do tamanho da semente na acumulação de biomassa e nutrientes e na produtividade do feijoeiro. Pesquisa Agropecuária Brasileira, 37(12):1711-1718, doi: https://doi.org/10.1590/ $\underline{\text { S0100-204X2002001200006 }}$

Silva, R.P., Corá, J.E., Furlani, C.E.A., Lopes, A. (2008) Efeito da profundidade de semeadura e de rodas compactadoras submetidas a cargas verticais na temperatura e no teor de água do solo durante a germinação de sementes de milho. Ciência e Agrotecnologia, 32(3): 929-937, doi: https://doi.org/10.1590/S1413

Silva, L.R., Pereira, M.J., Azevedo, J., Gonçalves, R.F., Valentão, P., Pinho, P.G., Andrade, P,B. (2013) Glycine max (L.) merr., Vigna radiata L. and Mendicago sativa L. sprouts: A natural source of bioactive compounds. Food Research International, 50(1):167-175, doi: https://doi. org/10.1016/j.foodres.2012.10.025

Steiner, F., Zuffo, A.M., Busch, A., Sousa, T.O., Zoz, T. (2019) Does seed size affect the germination rate and seedling growth of peanut under salinity and water stress? Pesquisa Agropecuária Tropical, 49, e54353, doi: https://doi. org/10.1590/1983-40632019v4954353

Vieira, R.F., Oliveira, V.R., Vieira, C. (2003) Cultivo do feijão-mungo-verde no verão em Viçosa e em Prudente de Morais. Horticultura Brasileira, 21(1): 37-43, doi: https://doi.org/10.1590/ S0102-05362003000100008

Vieira, R.F., Paula Júnior, T.J.P., Jacob, L.L., Lehner, M.S., Santos, J. (2011) Desempenho de genótipos de feijão-mungo-verde semeados no inverno na Zona da Mata de Minas Gerais. Revista Ceres, 58(3):402-405, doi: https://doi.org/10.1590/ S0034-737X2011000300022

Yu-Wei L., Wang Q. (2015) Effect of Processing on Phenolic Content and Antioxidant Activity of Four Commonly Consumed Pulses in China. Journal of Horticulture, 2(2):130-132, doi: https://doi. org/10.4172/2376-0354.1000130
Con icto de Intereses Los autores declaran no tener ningún con icto de intereses

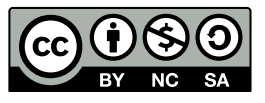

